\title{
Comunicación

\section{Notas para una exploración teórica sobre los estudios de producción de comunicación mediática (periodismo, opinión pública y comunicación política)}

En este texto se pretende explorar el sitio que ha ocupado el estudio de la producción de comunicación mediática y cómo ha sido armada en el contexto de la conformación e institucionalización del campo de estudio de la comunicación. Históricamente, se ha mantenido una preocupación fundamental por entender cómo las organizaciones mediáticas confluyen con otros actores sociopolíticos, y cómo los procesos de producción simbólica se conectan desde los medios con otros procesos económicos, políticos y socioculturales. ${ }^{2}$

PALABRAS CLAVE: campo de estudio, teorías de comunicación, opinión pública, periodismo.
This article looks for the place occupied by the studies of communication production in the communication field (and how these research works have been structured). Historically, it has had preoccupations in order to understand how the mass media are connected with sociopolitical actors, and how the symbolic production process from mass media has a link with the economical, political and sociocultural processes.

KEY WORDS: field of research, theories of communication, public opinion, journalism.

1 ITESO. Correo electrónico: sdeleon@correo.uaa.mx.

2 Dicha investigación se desarrolla como tesis en el programa de Doctorado en Estudios Científico-Sociales en el ITESO bajo el título todavía no definitivo de "Actores sociopolíticos y periodismo: prácticas y estrategias en la configuración de lo público-mediático". 


\section{INTRODUCCIÓN}

Este documento busca dar cuenta de la necesidad de construir una articulación ${ }^{3}$ que contribuya a configurar una postura sobre el estudio de la producción de comunicación mediática. Ubicarse en esas coordenadas implica detenerse a mirar el polo emisor del proceso y dar cuenta de los estudios, perspectivas teóricas y tradiciones de investigación que han analizado la producción y puesta en circulación de los mensajes mediáticos. Significa dar cuenta de las condiciones inherentes a los dispositivos de comunicación pública y las prácticas de los sujetos que con ellos se relacionan en un contexto urbano.

El gran interés por desplazar la discusión académica sobre la comunicación de las estructuras hacia los sujetos desde una perspectiva sociocultural, particularmente en América Latina, ha generado importantes aportes para pensar la producción mediática desde otros referentes: las interacciones de los sujetos y su correlato sobre la producción de sentido. Sin embargo, también parece haber tenido otra lectura: que indagar sobre los medios de comunicación es cosa del pasado. ${ }^{4}$ El trán-

3 Utilizo el término "articulación" para referirme a la elaboración de mapas teórico-metodológicos que permitan, por un lado, recuperar el conocimiento formulado con relación a la producción de comunicación mediática y, por otro, tomar puntos de referencia para situar (y también para descolocar) reflexiones y objetos de estudio así como modos de abordarlos. Esto implica, como lo sugiere Martín-Barbero (2002:205-255), la construcción de mapas y marcos de referencia de manera simultánea, es decir, la elaboración de cartografías.

4 Durante la sesión plenaria de conclusiones en el XVII Encuentro Nacional de Investigadores de la Comunicación organizado por la Asociación Mexicana de Investigadores de la Comunicación (AMIC), celebrado en la ciudad de Mérida, Yucatán del 19 al 21 de mayo de 2005, Tanius Karam señalaba como comentario al margen -al parecer asumiendo el marco de la "comunicología posible" (Galindo, 2004:127-145)-, la necesidad de dejar atrás el mediacentrismo y enfocar otros objetos de investigación relevantes en el estudio de la comunicación. En ese momento, Karam ponía el énfasis en el hecho de que por fin se habían eliminado mesas de trabajo como las de 
sito de los medios a las mediaciones no es una condición irreversible, Martín-Barbero (1993:300) lo propone simplemente como otra manera de ver, sin ser ella misma totalizadora.

Recientemente, Martín-Barbero (2001:15-42) ha propuesto una agenda de trabajo para el estudio de la comunicación que se genera a partir de las siguientes mediaciones comunicativas: socialidad, ritualidad, institucionalidad y tecnicidad. Cada una de ellas mantiene una esfera de articulaciones, pero al mismo tiempo se articulan entre sí. En su reflexión Martín-Barbero nos propone atender simultáneamente las dimensiones instrumentales y las ideológicas e intersubjetivas sustentadas en las primeras.

Esta propuesta aparece como coincidente con las formulaciones de la sociología reflexiva con su orientación estructural-constructivista (Bourdieu, 1988), que da cuenta de la importancia de atender tanto a las condiciones objetivas como a las subjetivas de los procesos sociales. En ese sentido, la consideración de estructuras y sujetos, sin soslayar uno ni otro, permite que la indagación en comunicación resulte estratégica

estudios de televisión, radio, cine -que siempre habían estado presentes-, para obligar a articular los estudios desde otros ejes temáticos. Se decía que la mesa de periodismo se mantenía pero se desestimaba en su carácter de investigación tradicional mediacentrista. En lo personal me encuentro en desacuerdo con esas aseveraciones y creo que persiste la necesidad de seguir preguntándose cosas acerca del periodismo y los medios. Es claro que hay que superar muchas ideas que parten de un pensamiento tradicional y conductista sobre la investigación de los medios de comunicación masiva. Sin embargo, el hecho de que se mantengan presentes en una sociedad que constantemente se transforma, nos hace pensar que ellos mismos, al formar parte de esa sociedad, se transforman también, por lo que se hace necesario hacer un esfuerzo creativo por entender esas transformaciones y cuestionarnos por ello. La investigación sobre medios está lejos de haberse agotado como lo demuestran, por ejemplo, el interés revisionista en los textos canónicos del campo de la comunicación, específicamente lo que se ha hecho sobre los medios, para entender que no son vetas agotadas de investigación y retomar rumbos en su estudio (Katz et al., 2003), así como la producción académica que en ese tenor sigue realizándose. 
para atender los fenómenos en toda su complejidad. Por lo anterior, vale la pena retomar la consideración de Graham Murdock (2002: 57) cuando señala que:

Para muchos analistas, incluido yo mismo, ilustrar el ejercicio del poder y sus condiciones estructurales y explorar las posibilidades para el cambio mantiene la pretensión central de una aproximación crítica sociocientífica a los medios y la comunicación. Como he tratado de indicar, en relación con esta tarea, tenemos un rico stock de conceptos y métodos para trabajar. Sus creadores no son figuras distantes que estén consignadas a las últimas salas en el museo de las ideas. Ellos permanecen como nuestros contemporáneos. Aún confrontamos las preguntas centrales que nos legaron, y su búsqueda de respuestas aún tiene mucho que enseñarnos. Somos parte de una conversación continua acerca de la estructura y el significado de los tiempos modernos y de las formas en que están cambiando. Ellos se paran en nuestros hombros avisándonos, criticándonos, urgiéndonos. Rehusar su invitación al debate es condenarnos a nosotros mismos a reinventar regularmente la rueda.

Es necesario explorar con perspectiva crítica la forma en la que se han estudiado los medios en México. La apuesta es la de articular una tradición que, por dispersa, no está lo suficientemente entendida. Es necesario armar una búsqueda que permita organizar la dispersión de la investigación sobre la producción de comunicación mediática para avanzar en una integración sistemática de este subcampo por la vía de su revisión.

En este documento se retoma el origen del interés por este tipo de estudios, vinculado al propio origen del campo de la comunicación. Posteriormente, se analizan algunas perspectivas teóricas que hacen énfasis en el proceso emisor de la comunicación mediática, y finalmente se señalan algunas ideas a manera de una conclusión inacabada (no puede ser de otra manera).

\section{LA PRODUCCIÓN DE COMUNICACIÓN MEDIÁTICA COMO PROBLEMA DE INVESTIGACIÓN}

En la actualidad, la comunicación como objeto de estudio convoca a la reflexión teórico-epistemológica a una cantidad importante de investi- 
gadores de las ciencias sociales y humanas al presentarse como un proceso complejo y multidimensional que es susceptible de ser analizado desde diversos ángulos, perspectivas teóricas y matrices disciplinarias.

Mientras que existen analistas que consideran que la comunicación debería ser objeto privilegiado de todas las ciencias sociales, ya que no existe ninguna dimensión de lo humano o lo social que no pueda entenderse mejor considerando los procesos comunicativos (Sánchez, 2002:2436 ), otros se muestran más cautelosos y señalan el riesgo de evitar que el enfoque de comunicación/cultura se convierta en una especie de efecto totalizador en el estudio de la sociedad y la cultura debido a que se ha puesto de moda $-\mathrm{y}$ como toda moda, puede ser efímera- en lugar de que el campo de la comunicación se transforme en un lugar legítimo desde el cual pensar la sociedad (Martín-Barbero, 2002:205-255).

Es interesante observar el desplazamiento del debate. En los años ochenta -y todavía entrada la década de los noventa-aún se luchaba por adquirir legitimidad y visibilidad en el contexto de las ciencias sociales. Ahora que por lo menos parece haberse alcanzado la segunda -aunque sea de manera parcial-, la preocupación de los agentes del campo de estudio de la comunicación parece enfocarse en la adquisición de densidad teórica crítica dentro del campo de las ciencias sociales en aras de afianzarse frente a los procesos de descentramiento disciplinario en un contexto de posdisciplinarización.

Las nuevas grandes narrativas de lo social ofrecidas por pensadores contemporáneos como Castells (2000:590), Giddens (1998:412) o Bourdieu (1999:232), donde se otorga centralidad a los procesos de comunicación, permiten contar con los marcos teóricos necesarios para redimensionar el lugar que la comunicación ha tenido en la ciencia social.

Es de tomarse en cuenta el hecho de que mientras se genera una visión crítica con respecto a las grandes narrativas de lo social y a la forma en la que las disciplinas científico-sociales han construido sus dominios bajo modelos decimonónicos carentes de toda vigencia en la actualidad (Wallerstein, 1999; 2004), el campo de la comunicación, por el contrario, aparece como un posible espacio transversal entre las disciplinas sociales. Las fronteras, origen y postulados de la comunicación son asumidos por los propios académicos interesados en su estudio 
como indeterminados, o en el mejor de los casos, como inter o transdisciplinarios (Sánchez, 2002:24-36 y Fuentes, 2002:12-29).

Desde las primeras tradiciones de la ciencia social moderna en las postrimerías del siglo XIX e inicios del XX, se introdujeron preguntas acerca de los fenómenos de la comunicación, principalmente como discusiones más bien centradas en los planteamientos epistemológicos, incluso ontológicos, que dirigieron la mirada de los científicos.

Quizás debido a que la comunicación es inherente a todas las actividades sociales, los propios fundadores del pensamiento social dieron la pauta para que se constituyera en elemento clave del estudio de la sociedad. Durkheim (1986:205), al hablar de las representaciones colectivas, y Weber (1983), al plantear la acción social provista de sentido, estaban construyendo ejes de análisis donde los fenómenos comunicativos tenían cabida de manera importante, aunque no se les llamara así explícitamente.

El posterior desarrollo de la tradición interpretativa -si seguimos a Lindloff (1995)- implicó un acercamiento de las ciencias sociales a las filosofías del lenguaje, a la filosofía pragmática y al pensamiento fenomenológico en la versión de Schutz. ${ }^{5} \mathrm{Al}$ consolidarse el estudio de lo social, en referencia a los procesos de significación intersubjetivos, la importancia del vínculo con la comunicación quedó de manifiesto.

La producción del sentido, en tanto resultado de un proceso de comunicación, pasa entonces a ser un objeto de interés de ciertos estudiosos que identificaron en ella la marca de la sociedad moderna a la que asistían, en la cual los dispositivos de comunicación de masas emergían potenciando los fenómenos de producción, circulación y consumo de signos e información, y con ello de las ideologías (Thompson, 1998).

El estudio de la comunicación de masas constituyó un campo emergente de investigación social. Se consideró que los medios representaban una fuerza a la vez política, económica y cultural. El interés por su análisis se presentó desde el principio de su proceso de industrialización. Fueron vistos como un escenario desde el cual explicar -con

5 Quien postulaba la idea de un mundo intersubjetivo anclado en las interacciones que los sujetos llevan a cabo, y a partir de las cuales se construye socialmente la realidad (Shutz, 2003:326). 
perspectiva comunicacional- diversos aspectos de la sociedad como el ejercicio del poder, la acumulación de capital o la construcción del acontecer público.

Posteriormente, el estudio de cómo se producen los mensajes desde los medios de comunicación emergió como un subcampo dentro del campo de estudios de la comunicación a partir de la segunda década del siglo XX, con las reflexiones y análisis de Walter Lippmann, John Dewey y otros (Carey, 1989). Esta situación se daba al mismo tiempo que se buscaba organizar disciplinariamente el estudio de comunicación, como sucedía con los esfuerzos de Harold D. Lasswell (1985) por integrar las áreas de análisis.

Probablemente fue Walter Lippmann (2003) quien, con la publicación de su obra Opinión Pública en 1922, presentó la forma fundacional más acabada de esta línea de trabajo. En el contexto de la escuela de pensamiento sociológico norteamericano, conocido genéricamente como la "Escuela de Chicago", Lippmann desarrolla este estudio centrado en el entendimiento del fenómeno de la opinión pública en la construcción de la vida democrática estadounidense. Da cuenta del papel central que se le otorga a los medios periodísticos como una garantía de libertad de opinión y expresión, situación imprescindible en una democracia. Por lo tanto, problematiza tal concepción analizando la forma en la que los medios construyen representaciones de lo público y las ponen a circular por medio de construcciones simbólicas de la realidad, como las noticias. También realiza abordajes de lo que representa el espacio público y el espacio privado.

Aunque no fue el primer libro que se escribió sobre los medios y su participación en las esferas públicas y privadas, en cambio sí constituyó el primer trabajo serio que confrontó a los medios de comunicación con marcos filosóficos y analíticos (Carey, 1989). Aún en la actualidad ese texto continúa debatiéndose y sugiriendo nuevas formulaciones para entender el vínculo entre los medios, la democracia, la esfera pública y la sociedad (Petersen, 2003:249-259; McCombs, 2006:290). Lippmann introdujo conceptos y categorías con las cuales todavía seguimos pensando muchos de los procesos de construcción de comunicación pública, como el hecho de que las noticias constituyen una reconstrucción social de la realidad. 
Las preguntas sobre la producción de comunicación mediática se orientaron específicamente hacia la producción periodística, con lo que el subcampo fue adquiriendo poco a poco una forma más definida. El pensamiento liberal del siglo XIX, que todavía permeó hasta las ideas de Lippmann, se manifestó en la construcción de una relación entre el periodismo y la democracia, esta última legitimada como la forma política moderna de las sociedades occidentales. Aún en la década de 1990 se "enfatiza que periodismo es otro nombre para democracia o, mejor dicho, que no puedes tener periodismo sin democracia" (Petersen, 2003: 249).

Una de las aportaciones principales de Lippmann fue la de problematizar el asunto de "la verdad" y "la información", para trasladarlos de una discusión ética a un asunto de representaciones de la realidad:

Él arguyó que un sistema libre de comunicación no garantizará información perfecta, y entonces no hay garantías de verdad aunque las condiciones de libertad sean seguras [... debido a] la naturaleza de las noticias y su recopilación, la psicología de la audiencia, y la escala de la vida moderna. Es importante notar lo siguiente: Lippmann redefinió el problema de la prensa como uno de morales y políticas a uno de epistemología" (Carey, 1989: 76).

Al considerar que existe un "mundo exterior e imágenes en nuestra cabeza", Lippmann (2003) también contribuyó a construir una teoría de las representaciones donde existen aspectos de la realidad que pueden ser construidos en forma de imágenes que organizamos en nuestras mentes y colocamos en ámbitos en los que esas imágenes se distribuyen. Esto también implica que existe una opinión pública en función de las mentes individuales que coinciden en las representaciones del mundo.

La idea anterior subsiste en las consideraciones modernas de la opinión pública. Vicent Price (1994) señala que en la actualidad la opinión pública puede ser definida por aquello que las encuestas diseñadas para el efecto pueden medir, lo cual tiene relación con el agregado estadístico de las representaciones construidas en mentes individuales al que hace alusión Lippmann.

John Dewey afinó un poco más esta idea al señalar que no es que existan representaciones sobre el mundo, a manera de imágenes que capturamos, sino que las representaciones son posibles gracias a la dis- 
cusión cuando la vida en comunidad es activa (Carey, 1989). Este autor iguala comunicación a la participación en la creación de un mundo colectivo, y por lo tanto, como el esencial problema político de la democracia (Peters, 1999).

En general, del pensamiento de Lippmann y Dewey - y podríamos agregar también a Robert Park y a Louis Wirth - se derivaron reflexiones y problematizaciones alrededor de conceptos como comunicación de masas, producción de noticias, opinión pública, consenso, representaciones de la realidad (Carey, 1989; Peters, 1999; Rothenbuhler, 2003:106-120), que mantendrán una tradición de indagación en el estudio de la comunicación alrededor de su producción y su vínculo con las esferas públicas.

Otras tradiciones también surgieron en torno al mismo objeto de estudio. Ya entrada la década de los 1930, Lasswell (1985) propone un modelo donde se sugieren cinco grandes áreas del estudio de la comunicación: 1) estudio de los emisores, 2) estudio de los mensajes, 3) estudio de los medios, 4) estudio de los receptores y 5) estudio de los efectos. Los tres primeros que implican el análisis del control (de cómo se fabrican los mensajes), el análisis del contenido de los mensajes y en análisis de los medios, se podrían relacionar estrechamente con un programa de investigación centrado en el proceso de producción de la comunicación mediática.

El mismo año en que se editó Opinión Pública, 1922, Lasswell ingresaba al departamento de ciencia política en la Universidad de Chicago, según Rodrigo Alsina (1989), por lo que no resulta desmedido suponer algún tipo de contacto académico entre ambos, pues compartieron el interés por analizar el papel de los medios periodísticos en la conducta política de la nación estadounidense.

Las contribuciones de Lasswell suelen ubicarse más bien en el subcampo del estudio de los efectos de los medios, debido principalmente a que su pensamiento se vio influenciado por el paradigma conductista y el periodo de la propaganda entreguerras. Sin embargo, sus aportaciones nutrieron a una tradición interesada en el estudio de los medios y sus productos desde una perspectiva funcionalista. Un ejemplo de ello son los estudios que integran la figura del gatekeeper (guardabarreras) en los procesos de selección de mensajes mediáticos. 


\section{PERSPECTIVAS TEÓRICAS RELACIONADAS \\ CON LA PRODUCCIÓN DE COMUNICACIÓN}

El estudio de la producción de comunicación mediática se ha desarrollado a lo largo de todo el siglo XX; sin embargo, no ha constituido todavía un subcampo sistemáticamente articulado. Los estudios que se han realizado desde el polo emisor de la comunicación se han caracterizado por una dispersión temática, epistemológica, teórica y metodológica. Vale la pena recuperar y tratar de articular esas visiones para contribuir a sistematizar el conocimiento que se ha construido al respecto.

Algunos autores han abierto la pauta para comenzar a realizar esa articulación. Por ejemplo Curran (1998:187-254) y Murdock (2002:4057) han elaborado un estado de la cuestión del campo de la comunicación en general, donde ponen énfasis especial en los abordajes de los medios de difusión desde las perspectivas de los estudios organizacionales de los medios, de economía política, de los estudios de las noticias y de la opinión pública. Así también están los proyectos más amplios, como el de McQuail (2001:632) y el de los esposos Mattelart (1997:142), que con la intención de historiar y organizar el campo han abordado las perspectivas teóricas relacionadas con la producción de comunicación, aunque no de manera exclusiva.

Este breve recorrido no intenta agotar las premisas bajo las cuales se ha investigado el fenómeno de la producción mediática. Antes bien, busca señalar algunos puntos de referencia para comenzar la articulación. Quizás se inicia desde lo más evidente, pero eso es necesario para llegar a lo más fino, que permita el diálogo entre perspectivas en función de los programas de investigación posibles alrededor de los procesos de producción mediática.

La presentación de las perspectivas teóricas que a continuación se enuncian tampoco está organizada de manera cronológica ni temática, sino más bien en función de intuiciones en proceso de sistematizarse, y pretenden que existe alguna conexión coherente entre una y otra.

\section{a) Teorías normativas de la prensa}

A partir de las recomendaciones formuladas por la comisión estadounidense de 1947 sobre la libertad de prensa, se propusieron una serie de 
premisas relacionadas con la responsabilidad social de los periodistas y los medios, pero también se estimularon los esfuerzos por definir las "teorías normativas de la prensa" (McQuail, 2001:632). Esto permitió establecer lineamientos de análisis de la producción de noticias de acuerdo con modelos periodísticos caracterizados por las teorías respectivas.

McQuail (idem) nos explica que en el foro que representó dicha comisión se discutieron elementos relacionados con la responsabilidad social que deben mantener los medios de comunicación cuyas obligaciones sociales equiparan el beneficio de su propiedad con un fideicomiso público. En ese tenor, se abordaron temas fundamentales para la construcción de una ética comunicativa que se relacionan con el respeto al ejercicio de las libertades de expresión y propiedad, así como con la idea de servicio público.

Se postularon cuatro teorías que corresponden a lo siguiente: teoría autoritaria, donde la prensa se sometía al control estatal; teoría libertaria, donde se asumía la libertad de expresión y de prensa como un elemento imprescindible en la práctica del periodismo; teoría totalitaria, donde el Estado controlaba de manera total a los medios periodísticos y se relacionaba directamente con los países soviéticos; 6 y la teoría de la responsabilidad social, ligada a la corriente del nuevo periodismo, donde los practicantes asumen una postura responsable frente a la elaboración de sus mensajes, en contraste con la teoría libertaria donde bajo la bandera de la libertad de prensa podían cometerse atropellos. A estas cuatro teorías se les adiciona posteriormente otras dos: la teoría del desarrollo, vinculada al deseable flujo internacional de noticias, sobre todo en países en vías de desarrollo que contribuyan a la libre determinación de los pueblos en lo económico y en lo político; y la teoría democrática-participativa, en la que se in-

6 Las implicaciones ideológicas y políticas son evidentes, pues si retomamos las propuestas de Lippmann, en el sentido de que es necesaria la democracia para la existencia del periodismo y viceversa, el postulado implica la enunciación "científica" de la inexistencia democrática en los países que conformaban el bloque socialista, y en ese sentido en conflicto con el estadio al que se aspira. 
corpora a los movimientos políticos y sociales que asumen una crítica cultural (Jensen, 2002:273-293).

Como puede observarse, estas teorías no son el resultado de un proceso de investigación empírica que mediante la confrontación de la realidad enuncien premisas que permitan explicarla o comprenderla. Son formulaciones construidas con dirección ideológica que actúan en el terreno del "deber ser" para legitimar determinadas prácticas de producción mediática y deslegitimar otras. Sin embargo, para comprender la preocupación por instalar la comisión de 1947, así como sus recomendaciones, es importante situarnos en el periodo histórico de fin de la Segunda Guerra Mundial, cuando la investigación académica sobre medios se preocupaba por los efectos inmediatos y la persuasión de la comunicación de masas en los diferentes públicos frente a problemas políticos de primera índole como la guerra, las relaciones internacionales y las elecciones nacionales. Tomar en cuenta ese contexto nos permite comprender la inquietud por establecer lineamientos de un correcto ejercicio de la producción periodística.

Estas teorías están conectadas con un pensamiento funcionalista derivado del pensamiento de Lasswell (1985), quien enuncia las funciones de los medios de comunicación como la vigilancia del entorno, la organización de la sociedad y la transmisión de la herencia social de una generación a la siguiente. El nexo se da particularmente con las dos primeras.

A pesar de que estas teorías normativas ya no se postulan por los investigadores académicos, François Demers (1998:59-84) ha propuesto su recuperación mediante un ejercicio de integración epistemológica al indicar que estas teorías normativas pueden trasladarse a herramientas metodológicas si se les aplica el método de los tipos ideales de Max Weber (1983), es decir, que pueden transitar de lo explicativo a lo comprensivo para dirigir la confrontación empírica, aun cuando varias de estas teorías han perdido legitimidad.

\section{b) El gatekeeping como proceso de selección}

En 1950 Manning White publicó un trabajo en el que describía la labor que realizaba Mr. Gates, un editor de un periódico local estadounidense, quien cumplía la labor de un gatekeeper (guardabarreras o portero), 
seleccionando información para ser publicada en forma de noticias. Existe coincidencia en señalar que el término gatekeeping fue acuñado por el psicólogo Kurt Lewin en un trabajo sobre los grupos familiares y cómo se tomaban las decisiones para la compra de comida (McQuail, 2001:632; Gomis, 1991:212).

El término gatekeeping se percibe no como una actividad de interés personal, sino como el resultado de las estructuras o esquemas organizacionales e ideológicos interiorizados en los individuos. Aplicado a los procesos de producción de comunicación mediática, el concepto tiene que ver con el control social en el interior de las redacciones periodísticas:

Inicialmente se prestó mucha atención al gran número de artículos que no lograban entrar y las razones de su rechazo. Estas investigaciones iniciales tendían a recalcar el carácter subjetivo de las decisiones de selección de noticias. Más adelante se prestó más atención a las influencias sistemáticas sobre la selección, que cabría considerar o bien "organizacionales" o bien "ideológicas". Las primeras se refieren principalmente a hábitos burocráticos, y las últimas, a valores e influencias culturales que no sean meramente personales o individuales, sino que surjan del entorno local (y nacional) de la actividad informativa (McQuail, 2001: 329).

A partir del estudio de White se desarrollaron un número importante de investigaciones para las cuales el papel del gatekeeper se convirtió en central (Gomis, loc.cit), situación por la que considero importante incluir en esta búsqueda de articulaciones este concepto como un eje destacado de la producción de comunicación.

En la actualidad, y en gran medida influenciados por las perspectivas de la sociología del periodismo (McNair, 1998) y del establecimiento de la agenda (McCombs, 2006:290), los estudiosos han dejado de usar este término sustituyéndolo por el de "selección noticiosa", que implica un proceso rutinario organizacional más armado y controlado.

Williams y Delli observan cambios en el proceso de gatekeeping en los medios estadounidenses, particularmente en la cobertura del caso de escándalo sexual Clinton-Lewinsky. Señalan los autores que "el escándalo Clinton-Lewinsky ilustra un cambio fundamental en el ambiente 
contemporáneo de los medios: la virtual eliminación del gatekeeping del flujo de la prensa" (2000: 61), o quizás ese proceso transita de los editores a los reporteros mismos, que han interiorizado fuertemente los criterios organizacionales del medio para el cual trabajan.

En un contexto donde las informaciones aparecen de manera tan rápida y súbita, los periodistas se ven en la necesidad de tomar decisiones de manera igual de rápida en la cobertura, sin la mediación de los sujetos que dominan las políticas organizacionales a las cuales debe ser sometida la producción de las noticias. Esto es particularmente cierto para el caso de los medios electrónicos, en los que un valor agregado de la cobertura periodística es la inmediatez de la información. Pero es claro que se trata de eventos extraordinarios. En la cotidianidad de la producción periodística las prácticas rutinarias están centradas en el control organizacional de la selección noticiosa (McNair, 1998; Esser, 1998).

\section{c) Economía política}

Desde una perspectiva crítica se abrió paso la corriente de la economía política. En ella, los pensadores cuestionan la forma en la que se ha configurado el mundo moderno capitalista, integrando un Nuevo Orden Mundial de la Información y la Comunicación (NOMIC) al servicio de un nuevo imperialismo: el cultural, lo que a su vez genera una dependencia cultural vertical entre países y entre sectores sociales (Mattelart y Mattelart, 1997:142).

En este sentido, la información y el conocimiento se distribuyen a través de las industrias culturales, las cuales tienen una conformación capitalista, y como tales las leyes del mercado y el interés por la acumulación del capital son las que dirigen las actividades de producción, distribución y consumo de la información.

Al mismo tiempo, las industrias culturales mantienen intereses políticos, pues el manejo de la información las vincula con las esferas del poder que controlan y ordenan los flujos informativos, que muy lejos de ser libre y horizontal es desequilibrado, vertical y presenta grandes brechas entre las regiones más ricas y las más pobres del mundo, como ya lo apuntaba la comisión dirigida por Sean McBride en su célebre informe presentado ante la UNESCO en 1980 (McBride, 1987:269).

McQuail (2001: 140) nos indica que la economía política 
Orienta la investigación hacia el análisis empírico de la estructura de propiedad y de control de los medios de comunicación, así como la manera en que operan las fuerzas de los mercados mediáticos. Desde este punto de vista conviene considerar la institución mediática como una parte del sistema económico estrechamente vinculada al sistema político.

Así, una dimensión concreta para el análisis de la producción de comunicación tiene que ver con la indagación sobre los estilos de la propiedad de los medios de comunicación. Preguntarse por la propiedad de los medios es preguntarse también por el control de la información y los mensajes. Y ese control tiende a ser cada vez más unilateral y concentrado.

En Estados Unidos, por ejemplo, se ha encontrado que existe una tendencia dominante que impone la concentración de la propiedad en manos de un número menor de compañías. Esta concentración se ha dado en función de cuatro tendencias principales: 1) la concentración de la propiedad, esto es, una gran cantidad de medios del mismo tipo que son propiedad una sola corporación; 2) la propiedad de medios cruzados, compañías que poseen distintos tipos de medios; 3) la propiedad conglomerada, compañías mediáticas que se involucran en otro tipo de negocios, como los deportes o los parques de diversiones; y 4) la integración vertical, compañías que controlan varios aspectos de la industria, como la producción y distribución de productos culturales (Biagi, 1999).

Murdock (2002:40-57) señala que existían críticos que denunciaban el papel de los medios periodísticos en el control sobre la democracia política, porque

los periódicos eran propiedad privada y entonces, estaban abiertos a abusos de poder por los propietarios a favor de sus opiniones o aquellos que eran sus aliados o asociados.

Esto se relaciona, en la esfera política, con los diversos bloques del poder que tienen acceso a los medios de comunicación, y en la económica con los anunciantes que influyen notablemente sobre la producción periodística (McNair, 1998).

En México, Enrique Sánchez (2001:71-111 y 2004:9-36), entre otros autores, ha contribuido a reflexionar sobre esta situación desde 
una perspectiva latinoamericana, abordando la dialéctica entre la realidad de la concentración de la propiedad de las industrias culturales y la necesidad de expansión e integración de ellas en la región latinoamericana para un intercambio informativo más horizontal, plural y verdaderamente mundializado.

\section{d) Media sociology y los estudios de la producción de noticias}

Parte de la preocupación por la manera en la que el estilo de la propiedad de los medios de comunicación influye en la producción de los mensajes, fue recogida también por una tendencia de investigación que surgió durante los años setenta. En ella se buscaba establecer cuáles eran las influencias que permitían que los mensajes de los medios fueran construidos bajo ciertos esquemas de producción y no otros.

La perspectiva se integró como un análisis de las rutinas de producción a partir de tres niveles: 1) ocupacional, donde se estudian las características individuales de los sujetos que trabajan en la producción de mensajes en los medios en relación con la organización en que se insertan; 2) organizacional, donde se abordan las rutinas de producción que funcionan mediante una lógica interna de la organización, así como las relaciones entre los sujetos que ocupan diferentes posiciones en la empresa mediática; y 3 ) institucional o supraorganizacional, mediante el cual se analizan los nexos existentes entre las organizaciones mediáticas y otras organizaciones y sectores públicos o privados (Hirsch, 1980:265-284). Dimmick y Coit (1983:361-389) han reconocido hasta nueve niveles de análisis, haciendo una deconstrucción analítica de estos tres niveles expuestos.

Esta propuesta de estudio fue conocida como media sociology (Cervantes 1996:49-81) en tanto que buscaba ofrecer un estudio sociológico interpretativo de la producción mediática. Pero pronto se polarizó hacia el estudio de la producción de las noticias.

Emergió así la propuesta conocida como sociología del periodismo o sociología de la producción de noticias, que se orienta hacia el

análisis de las relaciones sociales e interacciones las cuales definen los parámetros de visión, el rango de autonomía, y la manera -a veces impuesta- en que (los periodistas) dan forma y contenido a lo que escriben y dicen acerca del mundo" (McNair, 1998:12). 
Una preocupación central de la sociología del periodismo fue la de dar cuenta de cómo las noticias son el resultado de un trabajo rutinario de recopilación, tratamiento y presentación de la información, y cómo estas rutinas están fundadas en la interiorización, por parte de los periodistas, de criterios organizacionales que conforman esquemas de interpretación del acontecer (Wolf, 1997; Hernández, 1995; De León, 2003:177).

En el proceso de la recopilación de la información, los periodistas tienen contacto con otros sujetos, por lo regular adscritos a alguna institución que los legitima como fuentes informativas para la prensa. Las relaciones entre los reporteros y sus fuentes han sido bastante analizadas para comprender cómo es que ciertos actores sociopolíticos tienen acceso a los medios noticiosos y otros no, y cómo en función de aquellos con los que se relacionan los periodistas se producen las noticias. Asimismo, se ha entendido que las fuentes informativas son organizadas por los medios noticiosos a manera de red, que constituyen ámbitos noticiales desde los cuales se puede acceder a información noticiosa (Sigal, 1973; Tuchman, 1983).

En la actualidad se estudia cómo han emergido nuevas prácticas de relaciones entre las fuentes informativas y los medios de comunicación, sobre todo aquellas orientadas por el modelo de las relaciones públicas, que han modificado de manera significativa la forma en la que las fuentes acceden a los medios (Davis, 2000:39-59).

Otro elemento importante se encuentra en la manera en la que los periodistas actúan en relación con la información y con las fuentes, es decir, los valores noticiosos. Mediante los criterios que los periodistas han interiorizado a partir de un proceso complejo de socialización y que además consensan con otros sujetos en el ejercicio cotidiano de su profesión, es posible que otorguen más interés a algunos acontecimientos con respecto a otros porque resultan más "noticiables". Ejemplos de valores noticiosos son la objetividad, la proximidad de un acontecimiento respecto al contexto de referencia, la actualidad de la información, la exclusividad, la notoriedad otorgada a una fuente informativa, entre otros (Gans, 1980:375 y Sigal, 1973).

Finalmente, la perspectiva incluye la investigación de la autopercepción de los periodistas y las representaciones que le otorgan a su 
propio trabajo. Los periodistas se perciben como sujetos determinados por múltiples circunstancias, como el tiempo y las condicionantes organizacionales. Al mismo tiempo, se ubican a sí mismos como intermediarios entre el gobierno y la gente, entre los políticos y los votantes, cuando en realidad son representantes de una empresa privada. Se consideran los defensores de la libertad de expresión y de opinión. Así, los periodistas se invisten a sí mismos con la capacidad de contribuir a la generación de opinión pública y, por lo tanto, a la construcción de la democracia que sintetiza todos los valores sociales a los que se aspira (De León, 2003:177).

En México, estudios de este tipo iniciaron a mediados de la década de 1980 con los trabajos pioneros de Gabriel González Molina (1986) y continuaron en la década de 1990 con los de Cecilia Cervantes (1996:49-81) y María Elena Hernández (1995) principalmente, ${ }^{7}$ así como las revisiones teóricas de José Carlos Lozano (1995). En la actualidad, el interés por estudiar la producción de noticias en México ha crecido, principalmente a través de tesis de posgrado (Hernández, 2000), donde cabe destacar los trabajos producidos en los programas de posgrados de la Universidad de Guadalajara y del Instituto Tecnológico y de Estudios Superiores de Monterrey (ITESM), campus Monterrey, en los cuales esta línea de estudio aparece más consolidada.

\section{e) Producción de comunicación social}

Desde un punto de vista histórico-estructural, Manuel Martín Serrano (1994:331) sugiere que todas las formaciones sociales, a través de la historia de occidente, han tenido un Sistema Social (SS) en interdependencia con un Sistema de Producción de Comunicación (SC). Esta interdependencia implica, además, autonomía de los dos sistemas, por lo que no se puede concluir que las transformaciones en el sistema de comunicación correspondan "mecánicamente" con las transformaciones en el sistema social.

7 Estos autores tienen una numerosa cantidad de trabajos publicados. En esta ocasión tan sólo se cita un ejemplo representativo de la labor que han desarrollado. 
El SS está organizado para manejar y transformar materias, energías e información con vistas a la satisfacción de las necesidades individuales y colectivas (biológicas, sociales, espirituales) de los miembros de la sociedad [mientras que el SC] se organiza para manejar esos mismos elementos, pero su referencia específica son los aconteceres, es decir, aquello que sucede o que deja de suceder y afecta a la comunidad (Martín Serrano, 1994: 53).

Ambos sistemas permanecen en afectación mutua respecto a sus componentes, relaciones estructurales y funcionales internas.

La propuesta, entre otras cosas, señala que existe un modo específico de producción de comunicación pública cuando una formación social se apropia de la información pública. Esto implica que existen condiciones sociohistóricas a partir de las cuales una sociedad ha institucionalizado el uso de la información pública, y en los diferentes momentos históricos, los modos de producción de comunicación pública han sido diferentes.

La comunicación pública requiere de un orden institucional específico que se corresponde con la necesidad de: a) organizar su actividad, b) tratar la información, c) identificar los temas que le competen, d) mantener rituales de elaboración de información pública, y e) identificar a sus actores.

A pesar de que esta propuesta tiene un enfoque primordialmente estructural, se observan componentes relativos (aunque no explícitos) a la acción de los sujetos en la manera en la que el autor señala que se genera la apropiación de la comunicación pública (o lo que es lo mismo, su modo de producción) en las formaciones capitalistas contemporáneas: a través de la producción del acontecer público.

Denomino "acontecer público" a cualquier emergente (cosa, objeto, suceso, observación, idea, norma, relación, etc.) que por su presencia o ausencia afecta o puede afectar a la comunidad y cuyo conocimiento puede ser compartido por los miembros de aquélla, porque ha sido seleccionado como objeto de referencia por las instituciones que tienen a su cargo dar noticia pública de lo que acontece (ibid: 123).

En la formulación del acontecer público se toman en cuenta los aspectos macroestructurales como el SS y el SC a través del orden ins- 
titucional de la producción de comunicación pública, el cual también funciona como una mediación de lo macroestructural con lo cotidiano, es decir, con los emergentes que afectan a la comunidad y a los cuales se ha decidido seleccionar para dar noticia pública.

El contrarrelato de lo anterior es la presencia de otros aconteceres o emergentes (mucho mayores en número) que no han sido seleccionados en ese proceso, y que por lo tanto nunca serán visibles públicamente, o por lo menos en términos periodísticos. Por lo que la especificidad de la capacidad de selección de las instituciones de la comunicación pública es central en esta consideración.

No obstante el alcance amplio que ofrece esta aproximación al estudio de la producción de comunicación mediática, tiene como limitación la invisibilidad del sujeto junto con la sedimentación historizada de su vida cotidiana a partir de las vivencias sociales. Este proceso abstrae esa historicidad del sujeto a la actuación institucional de la producción de comunicación pública.

\section{f) Comunicación política}

La relación entre el estudio de la producción de comunicación y la política ha sido evidente desde la propia fundación del campo. Lippmann y Dewey, desde la perspectiva interpretativa, y Lasswell, desde la explicativa, se preocuparon por el papel de los medios en la vida política de la nación norteamericana.

Sin embargo, la comunicación y la política se relacionan de una manera tan compleja, que es difícil establecer un marco teórico coherente que condense las preocupaciones centrales de una perspectiva de comunicación política.

En una interpretación, la comunicación política aparece ligada a la reflexión de la filosofía política; en otra, como una práctica inherente a la actividad de la prensa, sobre todo si se considera desde el pensamiento liberal; y en otra interpretación, como las actividades que llevan a cabo los políticos para desarrollar su propaganda a través de los medios de comunicación definido también como marketing político (Aceves, 2005:39-66).

Quizás un elemento que articule los estudios sobre comunicación política tenga que ver con el concepto de opinión pública. Habermas 
(1999:352) ha elaborado una amplia relación histórico-crítica en la que analiza la formación de la opinión pública con anclaje en el desarrollo de una esfera pública ligada principalmente al modo de producción capitalista y a la actividad desarrollada por los periodistas como una extensión de la discusión entre ciudadanos de los temas de interés colectivo.

Alrededor del concepto de opinión pública se han desarrollado estudios que vinculan la actividad política de manera más amplia (es decir, no sólo aquella que llevan a cabo los políticos) con la actividad de la producción de comunicación mediática y las percepciones que se tienen del entorno. El trabajo de Trenz (2004:291-319), donde se busca articular la presencia de la esfera pública emergente en el proceso de integración europea a través de la cobertura periodística es un ejemplo interesante. En México han aparecido múltiples esfuerzos para realizar un análisis sobre la relación de la política y los medios como los trabajos de Raúl Trejo (2005:206) y Fátima Fernández (2002:193), entre otros. Sin embargo, desde las coordenadas de los estudios de producción de comunicación los casos son aislados. Un ejemplo es el trabajo de Aguilar (1998:84-125), donde se da cuenta de cómo el espacio mediático noticioso de la capital de la república constituye un verdadero espacio público complejo donde se verifica gran parte de la vida pública de la metrópoli.

\section{g) Establecimiento de la agenda}

La teoría del establecimiento de la agenda, o agenda setting, se vincula principalmente con el estudio de los efectos de los medios más que con la producción mediática (McCombs, 1996:13-34 y 2006:290; Wolf, 1997), pero tiene una dimensión que se conecta directamente con los estudios de producción de comunicación mediática. Se trata de la conformación temática de las noticias (selección noticiosa) y las fuentes de información alrededor de las cuales es producida.

La hipótesis de la agenda setting se construyó alrededor de la pregunta: ¿quién establece la agenda pública? Las investigaciones pioneras terminaron por concluir que los medios de comunicación no solamente ofrecen noticias, sino que contribuyen a construir categorías del acontecer sobre las cuales parecería importante pensar (idem).

La clásica frase atribuida a la agenda setting nos indicaría entonces que los medios no nos dicen cómo pensar, sino que presentan los temas 
sobre los cuales es importante pensar y los invisten con atributos. Así, se va construyendo una agenda temática donde intervienen los medios de comunicación como proveedores, pero también la clase política y los públicos que van proponiendo otras temáticas hasta que finalmente alcanzan visibilidad en los medios. De tal manera que la afectación es bilateral no solamente de parte de los medios a los públicos, aunque se reconoce que los medios tienen un efecto directo, aunque no inmediato, sobre la priorización de los temas a través de la "orden del día" (idem).

Esta priorización de la orden del día, lejos de constituir el resultado de decisiones individuales en las salas de redacción noticiosa de los medios, nos habla de un proceso de larga sedimentación en el cual cotidianamente se establecen los temas de la agenda mediática en discusión con otras agendas (las de los públicos y las de los políticos, principalmente). Cada agenda otorga ciertos atributos a los acontecimientos sociales a partir de los cuales parecen más o menos relevantes ante la mirada pública. Esta interacción es similar a las "capas de una cebolla", donde la influencia se concentra hasta su visibilización pública a través de los medios informativos (McCombs, 2006:290).

En estas agendas en interacción, la agenda mediática es relevante porque es la que con mayor fuerza puede colocar los temas ante los públicos y existen algunos de ellos que por diferentes factores (historicidad propia de la sociedad en que emergen, su confluencia con proyectos políticos determinados, entre otras cosas), mantienen preponderancia en los medios y van sedimentando en la sociedad, y finalmente su cobertura mediática ejerce un efecto de mediano y largo plazo entre la opinión pública.

McCombs (1996:13-34), el fundador de la teoría, señala que en parte la agenda pública es establecida por las tradiciones, las prácticas y los valores del periodismo como profesión. A ello habría que sumar el espacio prioritario que los medios le otorgan a las fuentes de información más notables, como el caso del presidente de la nación.

Por su parte, Cervantes (1999:133-152) realiza una crítica a un recuento que hace McCombs de su propia teoría a veinte años de su formulación, donde coloca en una cuarta fase de trabajo el estudio de la producción de las noticias. La crítica gira en torno a que no reconoce a la sociología del periodismo como un área de investigación indepen- 
diente de la agenda setting, evitando así una posibilidad de interrelación en la cual pudieran enriquecerse ambas perspectivas.

En México, uno de los principales autores que han desarrollado análisis importantes y enfrentado debates teóricos y metodológicos para entender los procesos del establecimiento de la agenda es Francisco Aceves (1993:223-225).

\section{CONCLUSIÓN (INACABADA)}

La búsqueda por una articulación integradora del estudio de la producción de comunicación mediática es compleja. La diversidad de temas, objetos, teorías y metodologías, en pocas palabras, de enfoques, obliga a pensar en un trabajo de largo plazo para formularla.

Uno de los principales retos para intentar aproximaciones más complejas y problematizadas al estudio de la producción mediática nos exige contar con un andamiaje teórico-metodológico adecuado que dé cuenta de esa realidad compleja. Considero necesario el desarrollo de la creatividad científica que nos permita generar integraciones desde diferentes perspectivas teóricas con la finalidad de contar con los conceptos necesarios articulados coherentemente para dotar de sentido a una realidad que opera en múltiples dimensiones por sí misma y, como dice Martín-Barbero (2002: 29), poder "cambiar el lugar desde donde se formulan las preguntas".

Aun con un recorte metodológico muy delimitado, el proceso de problematizar la realidad para investigarla nos exige esta labor si de verdad queremos ganar en profundidad y en capacidad interpretativa. Otro tanto sucede con las estrategias metodológicas que debemos construir para acercarnos y hacer aprehensible la realidad social. Es importante contar con dispositivos metodológicos múltiples y potentes que trasciendan explicaciones reduccionistas y unidimensionales.

La propuesta no radica en uniformizar todo lo que tenga que ver con el proceso de emisión de los mensajes mediáticos. Por el contrario, con este ejercicio se sugiere que sería interesante armar un mapa que permita dar cuenta de los itinerarios diversos que alrededor de este fenómeno se han construido.

¿Cuál sería la contribución de un trabajo de esta naturaleza? Todo mapa sirve para situarse. En este caso, el documento responde a la nece- 
sidad de situar preguntas sobre el vínculo entre las prácticas de producción periodística y las prácticas de construcción de lo público.

Las propuestas teóricas abordadas en este documento me parecen muy relevantes, pero la sistematización del conocimiento acerca de la producción mediática que presentan, mantiene ópticas distintas en cada una, a veces incluso opuestas. Sin embargo, al generar integraciones, podemos complementar visiones y posturas. Por lo tanto, las estrategias de construcción de los objetos de estudio deben asumir el recorte teórico-metodológico con el fin de hacer abordable empíricamente el problema de investigación, pero también deben considerar la necesidad de ubicarse en espacios intersticiales que nos permitan la conexión de perspectivas teóricas que doten a nuestros objetos de mayores recursos intelectuales para su investigación.

La producción de comunicación mediática se presenta en una realidad cuya complejidad debe asumirse. Una realidad compleja, desde mi perspectiva, no puede ser abordada mediante una sola dimensión de explicación teórica. Así pues, reconocer las diferentes teorías que han sido construidas alrededor de la producción de comunicación otorga la posibilidad de decidir la forma de integrar una visión más completa para abordar su estudio.

La formulación de líneas convergentes entre ellas, me parece importante para avanzar en la complejización de los problemas de investigación. La cuestión fundamental para la cual es necesario encontrar una respuesta es: ¿cómo abordar esa integración? Definitivamente no puede ser única ni rígida. Deberá responder a las necesidades de cada planteamiento, con flexibilidad creativa, pero con una observación puntual del rigor científico y coherencia explicativa-interpretativa. Asimismo, la propia integración teórica debe llevarnos a la elección metodológica, también creativa y flexible, para hacer observables y consecuentes los conceptos a los que hayamos decidido acercarnos.

Como puede observarse, el asunto de confrontar la complejidad en los estudios de producción mediática es huidizo y volátil. Pero también es fundamental. Por lo pronto, hay que decir que esta discusión tiene muchos puntos de entrada y hasta ahora ninguno claro de salida, debido a que necesita de una discusión académica y organizada de quienes nos interesamos por la investigación sobre este tema. 


\section{Bibliografía}

Aguilar, M. A. (1998) "Espacio público y prensa urbana” en García Canclini (coord.) Cultura y comunicación en la Ciudad de México II. La ciudad y los ciudadanos imaginados por los medios. México: UAM- Iztapalapa, Grijalbo.

ACEVES, F. de J. (1993) "La influencia de los medios en los procesos electorales. Una panorámica desde la perspectiva de la sociología empírica", Comunicación y Sociedad, núm. 18-19. México: CEIC / Universidad de Guadalajara.

- (2005) "Marketing político y rentabilidad electoral: el caso del Distrito 10 de Jalisco en las elecciones federales de 2003", Comunicación y Sociedad, núm. 4, nueva época. México: DECS / Universidad de Guadalajara.

BIAGI, S. (1999) Impacto de los medios. México: International Thomson Editores.

BOURDIEU, P. (1999) Razones prácticas. Sobre la teoría de la acción. Barcelona: Anagrama.

- (1988). Cosas dichas. Buenos Aires: Gedisa.

CAREY, J. (1989) Communication as culture. Essays on media and society. New York \& London: Routledge.

CASTELLS, M. (2000) La era de la información: economía, sociedad y cultura. Vol I: La sociedad red. México: Siglo XXI.

CERVANTES, C. (1996) "Construcción primaria del acontecer y planeación de la cobertura informativa. Propuesta metodológica para su estudio". Comunicación y Sociedad, núm. 28. México: DECS, Universidad de Guadalajara.

- (1999) "La sociología de las noticias como vía para renovar la investigación en la línea de agenda setting: revisión de interpretaciones", Comunicación y Sociedad, núm. 36. México: DECS, Universidad de Guadalajara.

CURRAN, J. (1998) "Repensar la comunicación de masas" en Curran et al. (comps.) Estudios culturales y comunicación. Análisis, producción y consumo cultural de las políticas de identidad y el posmodernismo. Barcelona: Paidós.

DAVIS, A. (2000) "Public relations, news productions and changing patterns of source access in the british national media", Media, Culture \& Society, vol. 22(1). London: Sage Publications. 
DE LEÓN, S. (2003) La construcción del acontecer. Análisis de las prácticas periodísticas. México: UAA.

DEMERS, F. (1998) “'Teorías normativas de la prensa' y 'tipos ideales' para interpretar los discursos sobre el periodismo", Comunicación y Sociedad, núm. 34. México: Universidad de Guadalajara.

DIMMICK, J. y P. Coit (1983) "Levels of analysis in mass media decision making. A taxonomy, research strategy and ilustrative data analysis", Mass Communication Review Yearbook, vol. 4. Beverly Hills: Sage.

DURKHEIM, Émile (1986) Las reglas del método sociológico. México: Fondo de Cultura Económica.

ESSER, F. (1998) "Editorial structures and work in british and german newsroom", European Journal of Communication, vol. 13, núm. 3. Reino Unido: Sage Publications.

FERNÁNDEZ, F. (2002) La responsabilidad de los medios de comunicación. México: Paidós.

FISHMAN, M. (1983) La fabricación de la noticia. Buenos Aires: Ediciones Tres Tiempos.

FUENTES, R. (2002) "Comunicación, cultura y sociedad: fundamentos conceptuales de la posdisciplinariedad", $\operatorname{Tram}(p)$ as de la comunicación y la cultura, núm. 1.

GALINDO. J. (2004) "Sobre comunicología y comunicometodología. Primera guía de apuntes sobre horizontes de lo posible", en Lennin Martell (coord.) Hacia la construcción de una ciencia de la comunicación en México. Ejercicio reflexivo 1979-2004. México: AMIC.

GANS, H. J. (1980) Deciding what's news. A study of CBS News, NBC Nightly News, Newsweek and Time. New York: Vintage Books.

GIDDENS, A. (1998) La constitución de la sociedad. Bases para la teoría de la estructuración. Buenos Aires: Amorrortu Editores.

GOMIS, L. (1991) Teoría del periodismo. España: Paidós.

GONZÁLEZ Molina, G. (1986) "Valores noticiosos: la distribución desigual del acceso periodístico", Avances de Investigación, núm. 3. México: Universidad de Colima.

HABERMAS, J. (1999) Historia y crítica de la opinión pública. La transformación estructural de la vida pública. España: Gustavo Gili.

HERNÁNDEZ, M.E. (1995) La producción noticiosa. México: CUCSH, Universidad de Guadalajara. 
- (2000) "La investigación sobre producción de noticias desde América Latina" en Orozco (coord.) Lo viejo y lo nuevo. Investigar la comunicación en el siglo XXI. Madrid: Ediciones de la Torre.

HIRSCH, P. (1980) "Occupational, organizational and institutional models in mass media research. Toward and integrated framework" Mass Communication Review Yearbook, vol. 1. Beverly Hills: Sage.

ISLA, L. (2002) "Desarrollo de la comunicación política" en Razón y Palabra, núm. 27 http://www.razonypalabra.org.mx/anteriores/n27/ lisla.html. México: ITESM.

JENSEN, K.B. (2002) "The social origins and uses of media and communication research", en Jensen (ed.) A handbook of media and communication research. Qualitative and quantitative methodologies. London \& New York: Routledge.

KATZ et al. (2003) Canonic texts in media research. Cambridge UK: Polity Press.

LASSWELL, H. (1985) "Estructura y función de la comunicación en la sociedad" en Moragas, Sociología de la Comunicación de Masas II. Barcelona: Gustavo Gili.

LINDLOFF, Thomas R. (1995) Qualitative communication research methods. Current communications: an advanced text series, vol. 3. USA: Sage Publications.

LippManN, W. (2003) Public Opinion. USA: University of Virginia American Studies, Program 2002-2003. http://xroads.virginia. edu/ Hyper2/CDFinal/Lippmann/contents.html (Versión electrónica).

LOZANO, José Carlos (1995) Teoría e investigación de la comunicación de masas. México: Editorial Alhambra Mexicana.

MARTÍN Serrano, M. (1994) La producción social de comunicación. México: Alianza Editorial.

Martín-BARbero, J. (1993) De los medios a las mediaciones. Comunicación, cultura y hegemonía. México: Gustavo Gili.

- (2001) "Deconstrucción de la crítica: nuevos itinerarios de la investigación”, en Vasallo y Fuentes (comps.) Comunicación. Campo y objeto de estudio. Perspectivas reflexivas latinoamericanas. México: ITESO, U de G, UAA, U. de Colima. 
- (2002) Oficio de cartógrafo. Travesías latinoamericanas de la comunicación en la cultura. Santiago de Chile: Fondo de Cultura Económica.

MATTElarT, A. y M. Mattelart (1997) Historia de las teorías de la comunicación. España: Paidós.

MCBRIDE, S. (1987) Un solo mundo, voces múltiples. Comunicación e información en nuestro tiempo. México: Fondo de Cultura económica.

MCCOMBS, M. (1996) “Influencia de las noticias sobre nuestras imágenes del mundo", en Bryant y Zillman (coords.) Los efectos de los medios de la comunicación. Investigaciones y teorías. Barcelona: Paidós.

- (2006) Estableciendo la agenda. El impacto de los medios en la opinión pública y en el conocimiento. España: Paidós.

MCNAIR, Brian (1998) Sociology of Journalism, London \& New York, Arnold Publishers. Co-published in USA by Oxford: University Press Inc.

MCQUAIL, D. (2001) Introducción a la teoría de la comunicación de masas. México: Paidós.

MURDOCK, G. (2002) "Media culture and modern times: social sciences investigations", en Jensen (ed.) A handbook of media and communication research. Qualitative and quantitative methodologies. London \& New York: Routledge.

PETERS, J.D. (1999) Speaking into the air. A history of the idea of communication. Chicago \& London: The University of Chicago Press.

PETERSEN, J. H. (2003) "Lippmann revisited. A comment 80 years subsequent to "Public Opinion", Journalism. Theory, practice \& criticism, vol. 4(2). London: Sage Publications.

PRICE, V. (1994) Opinión pública. México: Universidad de Guadalajara.

RODRIGO Alsina, M. (1989) Los modelos de la comunicación. España: Tecnos.

ROTHENBUHLER, E.W. (2003) "Community and pluralism in Wirth's 'consensus and mass communication", en Katz et al. (eds.) Canonic texts in media researchs. Cambridge: Polity Press.

SÁNCHEZ, E. (2001) "Integración latinoamericana e industrias culturales, dialéctica de la mundialización: un punto de vista histórico- 
estructural", Comunicación y Sociedad, núm. 39. México: DECS, Universidad de Guadalajara.

- (2002) "La investigación latinoamericana de la comunicación y su entorno social: notas para una agenda", en Diá-logos de la Comunicación núm. 64. Lima: FELAFACS.

- (2004) "El empequeñecido cine latinoamericano y la integración audiovisual... ¿Panamericana?: ¿Fatalidad de mercado o alternativa política?", Comunicación y Sociedad, núm. 2, nueva época. México, DECS: Universidad de Guadalajara.

SCHUTZ, A. (2003) El problema de la realidad social. Buenos Aires: Amorrortu.

SIGAL, Leon V. (1973) Reporteros y funcionarios. La organización y las normas de la elaboración de noticias. México: Ediciones Gernika, colección Política y Comunicación.

THOMPSON, J. (1998) Ideología y cultura moderna. Teoría crítica social en la era de la comunicación de masas. México: UAM Xochimilco.

TRENZ, H-J. (2004) "Media coverage on european governance. Exploring the european public sphere in national quality newspapers", European Journal of Communication, vol 19(3). London: Sage Publications.

Trejo, R. (2005) Poderes salvajes. Mediocracia sin contrapesos. México: Cal y Arena.

TuCHMAN, G. (1983) La producción de la noticia. México-BarcelonaBuenos Aires: Gustavo Gili S.A.

WALLERSTEIN, I. (1999) Impensar las ciencias sociales: límites de los paradigmas decimonónicos. México: Siglo XXI.

- (2004) The uncertainties of knowledge. Philadelphia: Temple University Press.

WEBER, Max (1983) Economía y sociedad. México: Fondo de Cultura Económica.

Williams, B.A. y M. X. Delli Carpini (2000) "Unchained reaction. The collapse of media gatekeeping and the Clinton-Lewinsky scandal", Journalism. Theory, practice \& criticism, vol. 1(1). London: Sage Publications.

WOLF, Mauro (1997) La investigación de la comunicación de masas. Crítica y perspectivas. México: Instrumentos Paidós. 